\title{
Design of an Error Model for Performance Enhancement of MEMS IMU-Based GPS/INS Integrated Navigation Systems
}

\author{
Moonsuk Koo', Sang Heon $\mathrm{Oh}^{2}$, Dong-Hwan Hwang ${ }^{1 \dagger}$ \\ ${ }^{1}$ A Department of Electronics Engineering, Chungnam National University, Daejeon 305-764, Korea \\ ${ }^{2}$ Hanyang Navicom Co., Ltd., Gwanpyeong-dong 713, Yuseong-gu, Daejeon 305-509, Korea
}

\begin{abstract}
In this paper, design of an error model is presented in which the bias characteristic of the MEMS IMU is taken into consideration for performance enhancement of the MEMS IMU-based GPS/INS integrated navigation system. The drift bias of the MEMS IMU is modeled as a 1st-order Gauss-Markov (GM) process, and the autocorrelation function is obtained from the collected IMU data, and the correlation time is estimated from this. Prior to obtaining the autocorrelation function, the noise of IMU data is eliminated based on wavelet. As a result of simulation, it is represented that the parameters of error model can be estimated correctly only when a proper denoising is performed according to dynamic behavior of drift bias, and that the integrated navigation system based on error model, in which the drift bias is considered, provides more correct navigation performance compared to the integrated navigation system based on error model in which the drift bias is not considered.
\end{abstract}

Keywords: MEMS IMU, error model, denoising, autocorrelation, bias stability

\section{INTRODUCTION}

The NAVSTAR Global Positioning System (GPS) is a satellite navigation system that started to be developed by the U.S. Department of Defense in 1973 for the purpose of overcoming the limitations of local radio navigation system like OMEGA, LORAN, TACAN, etc. (National 1995) and is utilized as the representative Global Navigation Satellite System (GNSS) until now since the Fully Operational Capability has been declared in 1994 (Kaplan \& Hegarty 2006). The GPS originally used for military purposes is now not only used as a major navigation system for aircraft, ships, and land vehicles but also takes its place in a major infra system for almost all sectors including the mobile phones.

Although the GPS receiver has the advantage that it can provide an accurate and stable position, velocity and time information of users regardless of meteorological conditions at anytime and anywhere, it has the disadvantage that it cannot provide the navigation results when the signals are

Received Sep 28, 2012 Revised Oct 23, 2012 Accepted Oct 26, 2012

†Corresponding Author

E-mail: dhhwang@cnu.ac.kr

Tel: +82-42-821-5670 Fax: +82-42-823-5436 blocked by buildings in downtown, or there is an intentional or unintentional radio jamming. In addition, the generic GPS receiver cannot provide attitude information, and because the output rate of navigation information is low, it is difficult to be used independently for vehicles with high dynamics like aircraft or guided weapons. In order to supplement these disadvantages of GPS, lots of researches on integrated navigation system in which the GPS and the Inertial Navigation System (INS) are combined have been performed (Bezick et al. 2010).

The GPS/INS integrated navigation system calculates the navigation information from output of accelerometer and gyro of the IMU, and corrects the INS navigation errors with the aid of GPS navigation results in the integrated Kalman filter which is based on the error model of the INS. The INS navigation errors are caused by bias, misalignment, scale factor, output noise, etc. included in output of the IMU (Titterton \& Weston 2004). In order to correctly estimate the INS navigation errors, the errors should be included in the error model of the integration Kalman filter (Maybeck 1979, Zarchan \& Musoff 2005). Recently, as the semiconductor technology develops, a low-price and highperformance Micro-Elecro Mechanical System (MEMS)based IMUs are widely used. In order to obtain the highperformance navigation results, it is required to take into 
full consideration the errors of MEMS IMU in the integrated Kalman filter.

In general, since the bias is known to have the largest impact on navigation errors of the IMU (Maybeck 1979), the bias is selected as an estimation variable for the Kalman filter. The bias can be divided into random constant bias and drift bias. The random constant bias does not change after the power is on and the drift bias is correlated with time and has a specific variance (Hulsing 1998). In general, it is known that the IMU with a smaller random constant bias has a smaller drift bias compared to the IMU with a larger random constant bias.

In Table 1, specifications of a commercial Ring Laser Gyro (RLG) IMU and a MEMS IMU are shown.

As shown in Table 1, the MEMS IMU of AIS has a larger random constant bias (bias repeatability) and a larger drift bias (bias stability) compared to the RLG IMU of Honeywell. After the GPS signal is blocked in the GPS/INS integrated navigation system, the navigation errors are greatly affected by the estimated values of errors of the IMU before the signal is blocked. Since the Kalman filter performance for error estimation is affected by accuracy of system error model, a better navigation performance can be expected if an error model with the drift bias as well as the random constant bias is used for the MEMS-based IMU.

In this paper, for performance enhancement of the GPS/ INS integrated navigation system using the MEMS IMU, a design of error model with the drift bias is described. As for methods to determine the error model of drift bias of the IMU, the $1^{\text {st }}$-order Gauss-Markov (GM) process model and $1^{\text {st }}$ and higher order Autoregressive (AR) process model can be considered (Godha 2006, Noureldin et. al. 2009). The

Table 1. Specifications of commercial IMUs.

\begin{tabular}{llcc}
\hline \multicolumn{2}{c}{ Specification } & $\begin{array}{c}\text { Honeywell } \\
\text { HG1700AG58 }\end{array}$ & $\begin{array}{c}\text { AIS } \\
\text { SiIMU02 }\end{array}$ \\
\hline Gyroscope technology & RLG & MEMS \\
Gyroscope error $(1 \sigma)$ & Bias repeatability $(\% / \mathrm{h})$ & 1.0 & 50.0 \\
& Bias stability $(\% / \mathrm{h})$ & 1.0 & 8.0 \\
& Scale factor $(\mathrm{ppm})$ & 150.0 & 250.0 \\
& Misalignment $(\mathrm{mrad})$ & 0.5 & 0.3 \\
& Random walk $(\% / \sqrt{\mathrm{h}})$ & 0.042 & 0.16 \\
Accelerometer error & Bias repeatability $(\mathrm{mg})$ & 1.0 & 2.5 \\
$(1 \sigma)$ & Bias stability $(\mathrm{mg})$ & 1.0 & 4.0 \\
& Scale factor $(\mathrm{ppm})$ & 300.0 & 300.0 \\
& Misalignment $(\mathrm{mrad})$ & 0.5 & 0.3 \\
Weight $(\mathrm{kg})$ & Randomwalk $(\mathrm{m} / \mathrm{s} / \sqrt{\mathrm{h}})$ & 0.007 & 0.16 \\
Size $(\mathrm{mm})$ & & 0.9 & 0.21 \\
Power consumption & $(\mathrm{W})$ & $127(\mathrm{~d}) \times 77(\mathrm{~h})$ & $65.5(\mathrm{~d}) \times 35.5(\mathrm{~h})$ \\
\hline
\end{tabular}

drift bias of the SiIMU02 IMU in Table 1 is known to be fully modeled as the $1^{\text {st }}$-order GM process (AIS 2008). Therefore, in this paper, a result of designing an error model with the drift bias is presented using the $1^{\text {st }}$-order GM process.

The autocorrelation function is obtained from the IMU output data, and the correlation time is determined from the autocorrelation function, which is a parameter of the error model of drift bias represented by the $1^{\text {st }}$-order GM process. Prior to obtaining the autocorrelation function, the random constant bias is obtained by averaging the IMU output, and noise of IMU data is eliminate using the wavelet. Relationship between decomposition level of denoising and estimation performance of the model parameter is investigated. Through simulations, the results on evaluation of the GPS/INS integrated navigation system with the proposed error model are given.

\section{DESIGN OF SDINS ERROR MODEL WITH THE CHARACTERISTICS OF MEMS IMU}

\subsection{SDINS Error Model with the Drift Bias}

The $\psi$ angle error model for position, velocity and attitude is given in Eqs. (1-3) in the navigation frame (Benson, Jr. 1975, Goshen-Meskin \& Bar-Itzhack 1992).

$$
\begin{aligned}
& \delta \dot{\mathbf{P}}^{n}=-\boldsymbol{\omega}_{e n}^{n} \times \delta \mathbf{P}^{n}+\delta \mathbf{V}^{n} \\
& \delta \dot{\mathbf{V}}^{n}=\mathbf{f}^{n} \times \delta \boldsymbol{\Psi}^{n}-\left(2 \boldsymbol{\omega}_{i e}^{n}+\boldsymbol{\omega}_{e n}^{n}\right) \times \delta \mathbf{V}^{n}+\mathbf{C}_{b}^{n} \delta \mathbf{f}^{b} \\
& \delta \dot{\Psi}^{n}=-\boldsymbol{\omega}_{i n}^{n} \times \delta \boldsymbol{\Psi}^{n}-\mathbf{C}_{b}^{n} \delta \boldsymbol{\omega}^{b}
\end{aligned}
$$

where $\delta \mathbf{P}^{n}, \delta \mathbf{V}^{n}$ and $\delta \Psi^{n}$ denote positional error, velocity error, attitude error, respectively. $\mathbf{f}^{n}$ denotes acceleration vector. $\delta \mathbf{f}^{b}$ and $\delta \boldsymbol{\omega}^{b}$ denote error vector of accelerometer and gyro of IMU, respectively. $\mathbf{C}_{b}^{n}$ denotes the direction cosine matrix from the body frame $(b)$ to the navigation frame $(n) . \boldsymbol{\omega}_{i e}^{n}$ denotes to earth rate, $\boldsymbol{\omega}_{e n}^{n}$ craft rate, $\boldsymbol{\omega}_{i n}^{n}$ angular velocity of the navigation frame relative to the inertial frame ( $i$ ). If the errors of IMU is modeled as random constant bias and noise in Eqs. (2) and (3), they can be represented in Eqs. (4-7).

$$
\begin{aligned}
\delta \mathbf{f}^{b} & =\mathbf{b}_{a}+\boldsymbol{\eta}_{a} \\
\delta \boldsymbol{\omega}^{b} & =\mathbf{b}_{g}+\boldsymbol{\eta}_{g} \\
\dot{\mathbf{b}}_{a} & =0 \\
\dot{\mathbf{b}}_{g} & =0
\end{aligned}
$$

where $\mathbf{b}_{a}$ and $\mathbf{b}_{g}$ random constant bias of accelerometer and gyro, respectively, and $\boldsymbol{\eta}_{a}$ and $\boldsymbol{\eta}_{g}$ noise of accelerometer 
and gyro, respectively.

In Eqs. (4) and (5), considering the drift bias, it can be represented as Eqs. (8-11).

$$
\begin{aligned}
\delta \mathbf{f}^{b} & =\mathbf{b}_{a}+\delta \mathbf{b}_{a}+\boldsymbol{\eta}_{a} \\
\delta \mathbf{\omega}^{b} & =\mathbf{b}_{g}+\delta \mathbf{b}_{g}+\boldsymbol{\eta}_{g} \\
\dot{\mathbf{b}}_{a} & =-\frac{1}{\tau_{a}} \delta \mathbf{b}_{a}+\boldsymbol{\eta}_{b a} \\
\dot{\mathbf{b}}_{g} & =-\frac{1}{\tau_{g}} \delta \mathbf{b}_{g}+\boldsymbol{\eta}_{b g}
\end{aligned}
$$

where $\delta \mathbf{b}_{a}$ and $\delta \mathbf{b}_{g}$ denote drift bias of accelerometer and gyro, respectively, and $\tau_{a}$ and $\tau_{g}$ correlation time of accelerometer and gyro, respectively. $\boldsymbol{\eta}_{b a}$ and $\boldsymbol{\eta}_{b g}$ denote driving noise of accelerometer and gyro, respectively.

From Eqs. (1-4) and Eqs. (8-11), the SDINS error model in a state Eq. can be obtained as the following Eq. (12).

$$
\left[\begin{array}{c}
\dot{\mathbf{x}}_{n a v} \\
\dot{\mathbf{x}}_{I M U}
\end{array}\right]=\left[\begin{array}{cc}
\mathbf{F}_{11} & \mathbf{F}_{12} \\
\mathbf{0}_{12 \times 9} & \mathbf{F}_{22}
\end{array}\right]\left[\begin{array}{c}
\mathbf{x}_{n a v} \\
\mathbf{x}_{M M U}
\end{array}\right]
$$

where $\mathbf{x}_{n a v}, \mathbf{x}_{I M U}, \mathrm{~F}_{11}, \mathrm{~F}_{12}$ and $\mathrm{F}_{22}$ are given in the following Eqs. (13-17), respectively.

$$
\begin{aligned}
& \mathbf{x}_{n a v}=\left[\begin{array}{lllllllll}
\delta p_{N} & \delta p_{E} & \delta p_{D} & \delta v_{N} & \delta v_{E} & \delta v_{D} & \delta \psi_{N} & \delta \psi_{E} & \delta \psi_{D}
\end{array}\right]^{T} \\
& \mathbf{x}_{I M U}=\left[\begin{array}{llllllllllll}
b_{a x} & b_{a y} & b_{a x} & b_{g x} & b_{g y} & b_{g z} & \delta b_{a x} & \delta b_{a y} & \delta b_{a x} & \delta b_{g x} & \delta b_{g y} & \delta b_{g z}
\end{array}\right]^{2} \\
& \mathbf{F}_{11}=\left[\begin{array}{ccc}
\boldsymbol{\omega}_{e n}^{n} & \mathbf{I}_{3 \times 3} & \mathbf{0}_{3 \times 3} \\
\mathbf{0}_{3 \times 3} & 2 \boldsymbol{\omega}_{i e}^{n}+\boldsymbol{\omega}_{e n}^{n} & \mathbf{f}^{b} \\
\mathbf{0}_{3 \times 3} & \mathbf{0}_{3 \times 3} & \boldsymbol{\omega}_{i n}^{n}
\end{array}\right] \\
& \mathbf{F}_{12}=\left[\begin{array}{cccc}
\mathbf{0}_{3 \times 3} & \mathbf{0}_{3 \times 3} & \mathbf{0}_{3 \times 3} & \mathbf{0}_{3 \times 3} \\
\mathbf{C}_{b}^{n} & \mathbf{0}_{3 \times 3} & \mathbf{C}_{b}^{n} & \mathbf{0}_{3 \times 3} \\
\mathbf{0}_{3 \times 3} & -\mathbf{C}_{b}^{n} & \mathbf{0}_{3 \times 3} & -\mathbf{C}_{b}^{n}
\end{array}\right] \\
& \mathbf{F}_{22}=\left[\begin{array}{cc}
-\frac{1}{\tau_{a}} \mathbf{I}_{3 \times 3} & \mathbf{0}_{3 \times 3} \\
\mathbf{0}_{3 \times 3} & -\frac{1}{\tau_{g}} \mathbf{I}_{3 \times 3}
\end{array}\right]
\end{aligned}
$$

Observing Eqs. (12-17), it can be seen that the Kalman filter is $15^{\text {th }}$-order when only the random constant bias is considered and $21^{\text {st }}$-order when both the random constant bias and the drift bias are considered.

The proposed error model is applied to the Kalman filter of the loosely-coupled GPS/INS integrated navigation system of which measurements are positional error and velocity error. The measured model is given in Eqs. (18-20).

$$
\begin{gathered}
\mathbf{z}=\mathbf{H} \mathbf{x}+\mathbf{v}, \mathbf{v} \sim N(0, \mathbf{R}) \\
\mathbf{z}=\left[\begin{array}{llllll}
\delta p_{N} & \delta p_{E} & \delta p_{D} & \delta v_{N} & \delta v_{E} & \delta v_{D}
\end{array}\right]^{T} \\
\mathbf{H}=\left[\begin{array}{lll}
\mathbf{I}_{6 \times 6} & \mathbf{0}_{6 \times 15}
\end{array}\right]
\end{gathered}
$$

where $\mathbf{v}$ is the measurement noise with zero mean and covariance, $\mathbf{R}$.

\subsection{Parameter Estimation of the Drift-Bias Error Model Using the Denoising Technique}

The $1^{\text {st }}$-order GM process in Eqs. (10) and (11) is expressed in Eqs. (21).

$$
\dot{x}(t)=-\beta x(t)+w(t)
$$

where $\beta$ denotes reciprocal of the correlation time, $\tau$ and $x(t)$ the drift bias. $w(t)$ is the white Gaussian noise with mean zero and variance, $\sigma^{2}$.

Since $\tau_{a}$ and $\tau_{g}$ in Eqs. (10) and (11) are correlation time of the drift bias of accelerometer and gyro represented by the 1st-order GM process, respectively, these are $\tau$ $\mathrm{s}$ at which the autocorrelation value $R_{x x}(\tau)=\sigma^{2} e^{-\beta|\tau|}$ becomes $\sigma^{2} e^{-1}$ in Eq. (18) (Brown \& Hwang 1997). In order to obtain $\tau_{a}$ and $\tau_{g}$, the random constant bias, $b_{a}$ and $b_{g}$ and the output noise, $\eta_{a}$ and $\eta_{g}$ in Eqs. (8) and (9) should be eliminated. In order to do this, estimation of the error model parameters should be performed after storing enough output data in stationary. The time span for data storage depends on the correlation time of drift bias errors (Nassar 2003). The random constant bias can be obtained by taking the average value of the data.

The denoising technique using the wavelet can be applied to eliminate output noise. In order to eliminate the noise in the output, the output data is decomposed into levels using the wavelet conversion, and a proper threshold is set for the decomposed output data at each level. After eliminating the noise in the output data, output data without noise is obtained by synthesizing the decomposed output data in reverse order. The noise cannot be eliminated if enough decomposition levels are not taken and on the other hand, both noise and signal are eliminated together if excessive decomposition levels are taken. Therefore, when the wavelet is used, it is important to have a proper decomposition levels which may be dependent on the dynamic behavior of the output data. 


\section{PERFORMANCE EVALUATION}

In order to show efficiency of the SDINS error model with the characteristics of MEMS IMU, a computer simulation was performed. First, relationship between number of wavelet decomposition level and estimation of parameters in the drift-bias error model was investigated. And navigation performance was compared for a looselycoupled GPS/INS integrated navigation system when the integration Kalman filter is $15^{\text {th }}$ order and the integration Kalman filter is $21^{\text {th }}$ order.

In order to eliminate noise in the computer simulation, 'cmddenoise' function was used in MATLAB Wavelet Toolbox. As parameters in the function ' $\mathrm{db5}$ ' and 'soft' are selected for wavelet function and for threshold, respectively. The MATLAB GPS Toolbox from GPSoft was used for GPS navigation solution data.

\subsection{Estimation of the Drift-Bias Error Model Parameters}

In order to investigate relationship between wavelet decomposition level and estimation of the drift-bias error model parameter, IMU data with characteristics of SiIMU02 MEMS IMU in table 1 were generated. In order to obtain variance of the driving noise when generating data by computer, consider the following discrete Eq. (22) which is a discretized form of Eq. (21) using the $1^{\text {st }}$-order Euler method.

$$
x_{k+1}=(1-\beta \Delta t) x_{k}+w_{k} \Delta t
$$

where $x_{k}$ denotes the discrete drift bias, $w_{k}$ the white Gaussian noise and $\Delta t$ sampling time. Variance of $x_{k}$ can be obtained in Eqs. (23) and (24).

$$
\begin{aligned}
\operatorname{var}\left[x_{k+1}\right] & =\sigma^{2} \\
& =(1-\beta \Delta t)^{2} \operatorname{var}\left[x_{k}\right]+\Delta t^{2} \operatorname{var}\left[w_{k}\right] \\
& =(1-\beta \Delta t)^{2} \sigma_{x}^{2}+\Delta t^{2} \sigma_{w}^{2} \\
\sigma_{w}^{2} & =\left(\frac{2 \beta}{\Delta t}-\beta^{2}\right) \sigma_{x}^{2}
\end{aligned}
$$

where $\sigma_{x}^{2}$ denotes variance of $x_{k}$, and $\sigma_{w}^{2}$ variance of $w_{k}$. It can be seen that variance of the driving noise for computer simulation can be obtained the variance of drift bias errors, sampling time and correlation time in Eqs. (10) and (11).

Since drift bias errors of SiIMU02 were set as the $1^{\text {st }}$-order GM process with 20 second correlation time, variance of Eq. (24) can be obtained as in Eq. (25) using ' $\sigma_{x}=8 \%$ h, $\beta$
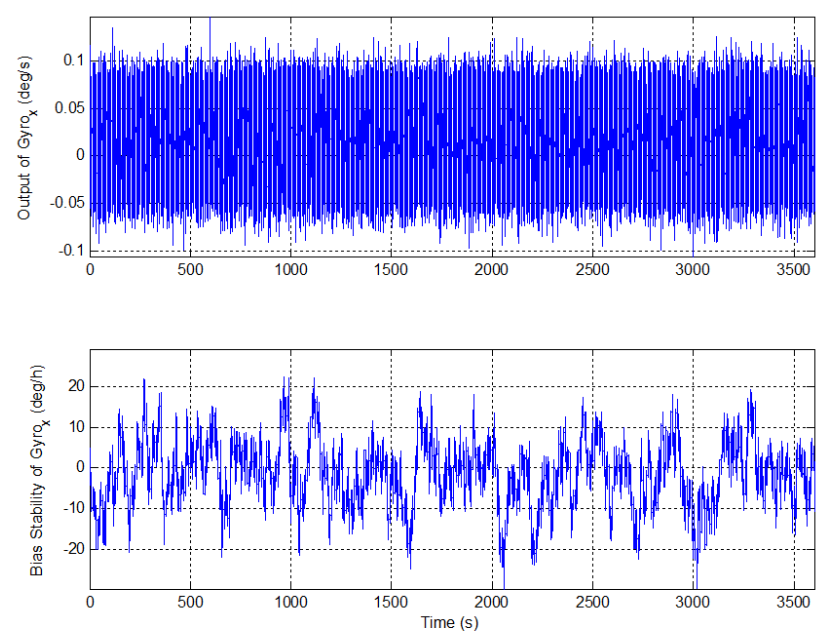

Fig. 1. Gyro output data in stationary for computer simulation.

$=1 / 20, \Delta t=0.01$ ' in Table 1 .

$$
\sigma_{w}^{2}=\left[\frac{2 \times \frac{1}{20}}{0.01}-\left(\frac{1}{20}\right)^{2}\right] \times 8^{2}[\circ / \mathrm{h}] \cong 25.3[\circ / \mathrm{h}]^{2}
$$

Fig. 1 shows gyro output result in stationary which is geneated using the variance Eq. (25). Average of gyro output, average of drift bias, and standard deviation of drift bias are $57.564 \% \mathrm{~h},-1.017^{\circ} / \mathrm{h}, 7.902 \% \mathrm{~h}$, respectively. Comparing these values with random constant bias of $50 \% \mathrm{~h}$, and drift bias of $8 \%$ h given in Table 1 , it can be seen that the gyro output data in Fig. 1 which is generated for computer simulation contain the characteristics of error of SiIMU02.

Fig. 2 shows autocorrelation functions of three wavelet decomposition levels of denoising for gyro output in Fig. 1. Fig. 2a is the autocorrelation function, $R_{x x}(\tau)$ for the true drift bias. Fig. 2b, c and $2 \mathrm{~d}$ are autocorrelation functions when decomposition levels are 5, 11 and 15, respectively. In figures, the correlation time is the value at which the autocorrelation value becomes $e^{-1} \cong 0.368$ since the autocorrelation function was normalized by variance. Table 2 shows correlation times to decomposition levels.

From Fig. 2 and Table 2, It can be seen that the correlation time is close to ' 0 ' due to noise when decomposition level is not enough and the correct correlation time is not able to be estimated when excessive levels are taken.

Fig. 3 shows relationship between correlation time and decomposition level. Estimated correlation time errors versus decomposition level are shown for gyro outputs with correlation time of drift bias 2 seconds, 20 seconds and 200 seconds, respectively. It can be seen that the best estimated 
(a) True Bias Stability

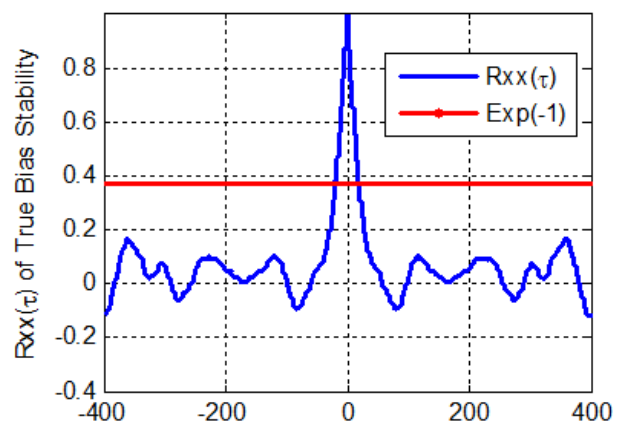

(c) Level 11

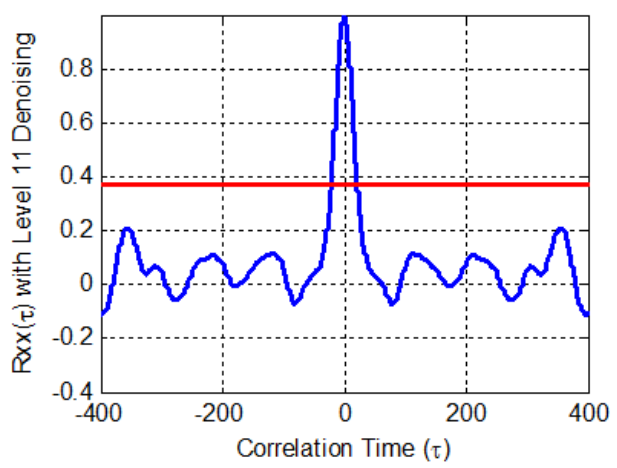

(b) Level 5

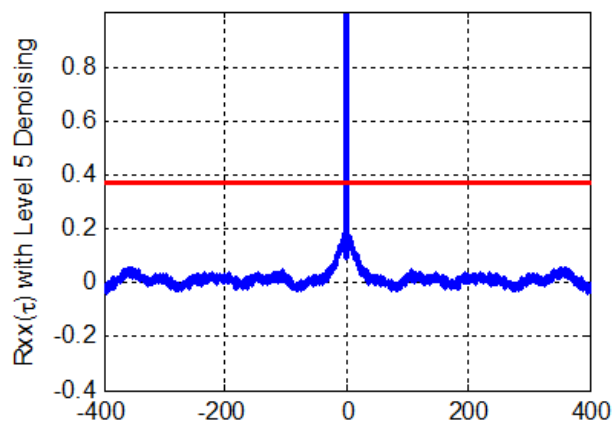

(d) Level 15

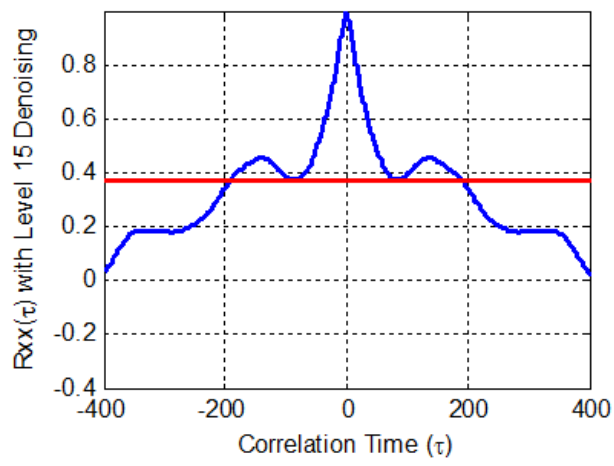

Fig. 2. Autocorrelation function (a) true bias, (b) level 5, (c) level 11, (d) level 15.

Table 2. Estimated values of correlation time versus noising level.

\begin{tabular}{cc}
\hline Denoising level & Correlation time $\tau$ (second) \\
\hline True value of drift bias & 20.0 \\
5 & 0.26 \\
11 & 21.5 \\
15 & 191.61 \\
\hline
\end{tabular}

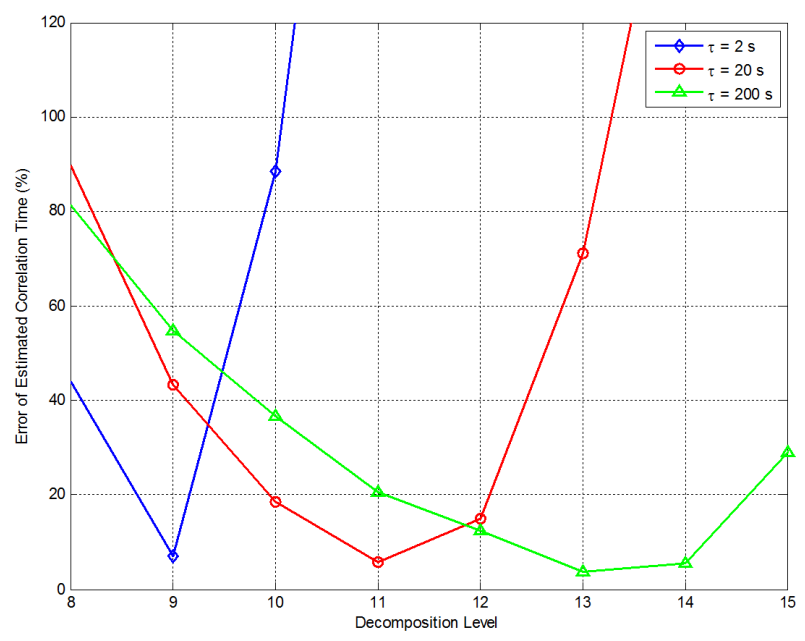

Fig. 3. Correlation time versus decomposition level.

performance is able to be obtained when the levels are 9, 11 and 13 level for gyro data sets, respectively. In addition to that, it can be seen that the higher decomposition level is required as the correlation time is the longer.

\subsection{Performance Evaluation of the MEMS IMU Error Model in the GPS/INS}

In order to evaluate the performance of MEMS IMU error model in the GPS/INS, a computer performed simulation was performed. Figs. 4 and 5 show reference trajectory of the simulation. The vehicle is stationary for 120 seconds, and moves with $9.8 \mathrm{~m} / \mathrm{s} / \mathrm{s}$ for 20 seconds toward $45^{\circ}$ northeast direction. After that, it climbs up toward ascends up to $4.8 \mathrm{~km}$ attitude for 115 seconds. And the vehicle performs a circular motion with $30^{\circ}$ bank angle. Finally, the vehicle descends to the ground. For the simulation of GPS, pseudorange data with $2.5 \mathrm{~m}$ RMS error and pseudo-range rate data with $0.05 \mathrm{~m} / \mathrm{s}$ RMS error were generated and processed these data using the least square method in order to generate navigation solutions. IMU data were generated using the error characteristics of the SiIMU02 IMU given in Table 1 and 3.1.

In Figs. 6, 7, and Table 3, the Root-Sum-Square (RSS) navigation errors for order of Kalman filter and estimated correlation time. The estimated correlation times are ' $\tau_{1}=0.26, \tau_{2}=21.50$ and $\tau_{3}=191.61$ ' to the decomposition 
levels in Table 2, respectively. GPS signal is blocked in the duration between 500 second and 560 second in the simulation. In Fig. 6 , it can be seen that both $15^{\text {th }}$-order Kalman filter and $21^{\text {st }}$-order Kalman filter gives similar navigation performance when GPS signals are available.

Fig. 7 and Table 3 show navigation errors in the duration when the GPS signal is blocked in Fig. 6. When the GPS signal is blocked, only INS is used in the navigation and the estimated bias just before the signal is blocked is used for correction of IMU errors. The $15^{\text {th }}$-order Kalman

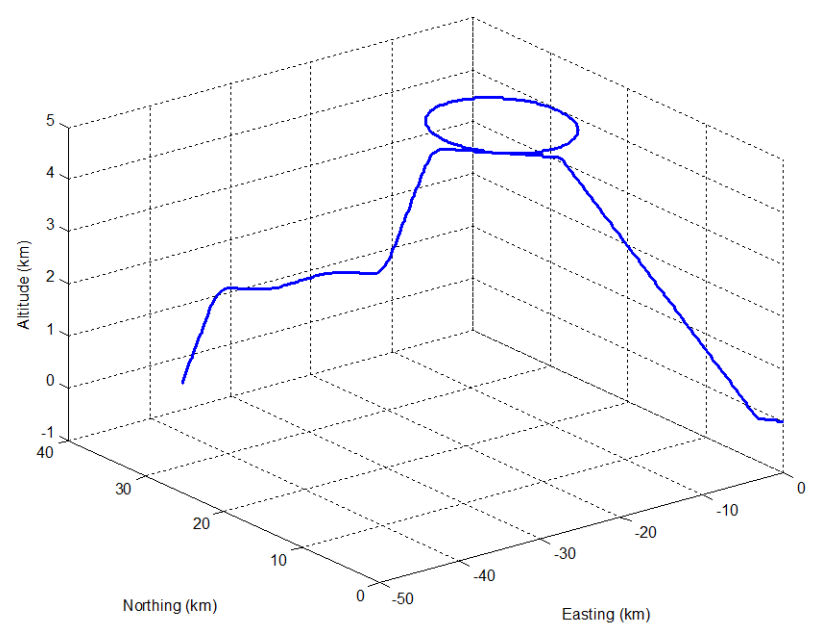

Fig. 4. Three-dimensional position reference trajectory.
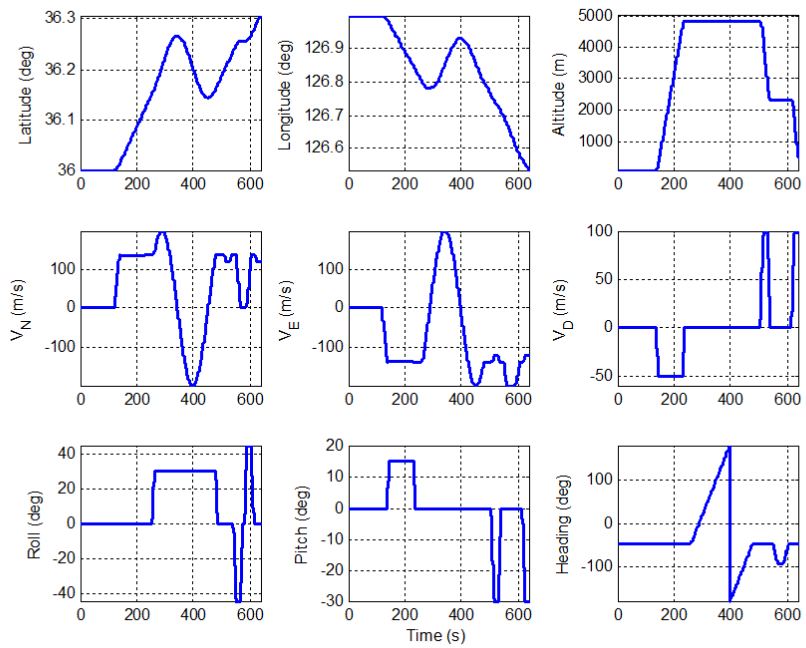

Fig. 5. Reference trajectory versus time. filter and the $21^{\text {st }}$-order Kalman filter with $\tau_{1}$ gives similar performance in Fig. 7 . It can be also observed that the $21^{\text {st }}$ order Kalman filter with $\tau_{1}$ gives a little bit less accurate navigation results. Comparing the result of the $21^{\text {st }}$-order Kalman filter with $\tau_{2}$ which is closest to true correlation time with that of the $21^{\text {st }}$-order Kalman filter with $\tau_{3}$, it can be seen that, attitude result of the $21^{\text {st }}$-order Kalman filter with $\tau_{2}$ are around 4 times more accurate than that of the $21^{\text {st }}$-order Kalman filter with $\tau_{3}$ although the position and velocity output are similar. From the simulation results,
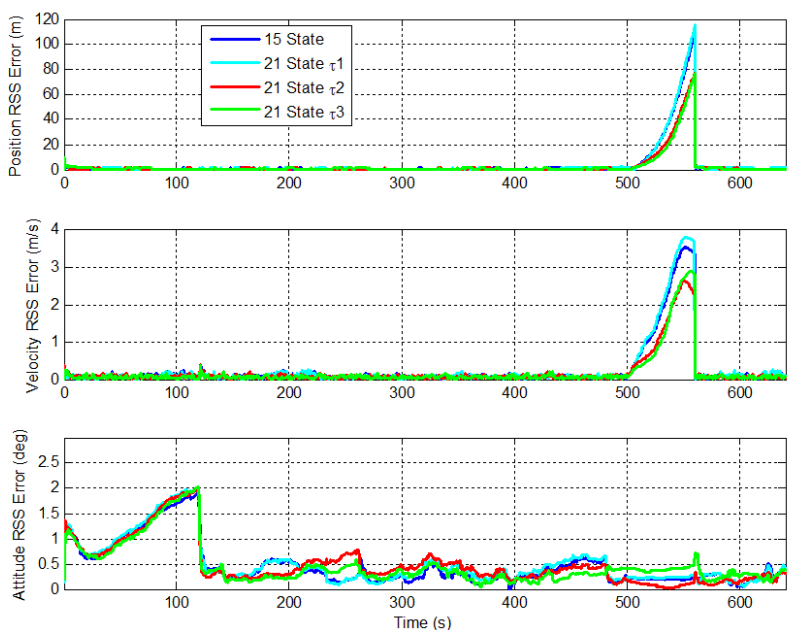

Fig. 6. Navigation error.
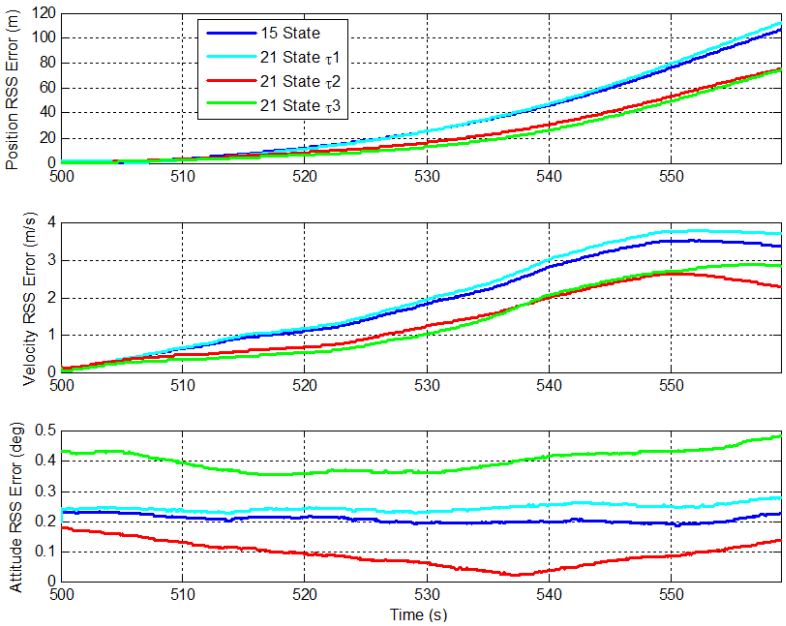

Fig. 7. Navigation errors when the GPS signal is blocked.

Table 3. Navigation errors when the GPS signal is blocked.

\begin{tabular}{lcccccccc}
\hline \multirow{2}{*}{ RSS errors } & \multicolumn{3}{c}{$15^{\text {th }}$} & \multicolumn{2}{c}{$21^{\text {st }} \tau_{1}$} & \multicolumn{2}{c}{$21^{\text {st }} \tau_{2}$} & \multicolumn{2}{c}{$21^{\text {st }} \tau_{3}$} \\
\cline { 2 - 9 } & Avg. & Max. & Avg. & Max. & Avg. & Max. & Avg. & Max. \\
\hline Position(m) & 34.57 & 106.34 & 35.46 & 111.89 & 23.90 & 75.14 & 21.33 & 74.27 \\
Velocity (m/s) & 1.90 & 3.52 & 2.04 & 3.78 & 1.35 & 2.64 & 1.32 & 2.88 \\
Attitude $\left({ }^{\circ}\right)$ & 0.21 & 0.23 & 0.25 & 0.28 & 0.09 & 0.18 & 0.40 & 0.48 \\
\hline
\end{tabular}


it can see be seen that the error model of the MEMS IMU with a drift bias gives a better navigation performance than error model with only a random constant bias that more excellent navigation performance is given when a more accurate parameters are estimated.

\section{CONCLUSIONS}

In this paper, results of designing an error model with characteristics of MEMS IMU have been presented in order to have performance enhancement of the GPS/INS integrated navigation system. The MEMS IMU errors have been divided into the random constant bias and the drift bias. The random constant bias was modeled as a random constant, and the drift bias as a $1^{\text {st }}$-order GM process. In order to estimate the correlation time which is a parameter of the 1st-order GM process, the autocorrelation function was used. Prior to obtaining the autocorrelation function, noise was eliminated based on wavelet. It has been shown that the parameters of model can be accurately estimated correctly only when a proper decomposition level is taken. Through computer simulations, It has been shown that an integrated navigation system gives a better navigation performance when a $21^{\text {st }}$-order Kalman filter with the MEMS error model is used.

Further studies will be carried out on a method to determine the decomposition level to get a better estimate of the parameter and on GPS/INS integration from real measurement output of MEMS IMUs.

\section{ACKNOWLEDGMENTS}

This research was supported by Basic Science Research Program through the NRF (National Research Foundation) of Korea funded by the Ministry of Education, Science and Technology (2012R1A1A2041839).

\section{REFERENCES}

AIS 2008, SiIMU02 Preliminary Product Specification for Precision Guided Munition (PGM) Application, Document No IMU02-22-0100-130 Revision 3 (Plymouth: AIS)

Benson, Jr., D. O. 1975, A comparison of two approaches to pure-inertial and doppler-inertial error analysis, IEEE Transaction of Aerospace and Electronic Systems, 11, 447-455
Bezick, S. M, Pue, A. J., \& Patzelt, C. M. 2010, Inertial navigation for guided missile systems, Johns Hopkins APL Technical Digest, 28, 331-342

Brown, R. G. \& Hwang. P. Y. C. 1997, Introduction to Random Signals and Applied Kalman Filtering, 3rd ed. (New York: John Wiley \& Sons)

Godha, S. 2006, Performance Evaluation of Low Cost MEMS-Based IMU Integrated With GPS for Land Vehicle Navigation Application, MS Thesis, Department of Geomatics Engineering, The University of Calgary

Goshen-Meskin, D. \& Bar-Itzhack, I. Y. 1992, Unified approach to inertial navigation system error modeling, Journal of Guidance, Control, and Dynamics, 15, 648653

Hulsing, R. 1998, MEMS inertial rate and acceleration sensor, IEEE Aerospace and Electronic Systems Magazine, 13, 17-23

Kaplan, E. D. \& Hegarty, C. J. 2006, Understanding GPS: Principles and Applications. 2nd ed. (Norwood: Artech House Inc.)

Maybeck, P. S. 1979, Stochastic Models, Estimations, and Control, vol.1 (New York: Academic Press, Inc.)

Nassar, S. 2003, Improving the Inertial Navigation System (INS) Error Model for INS and INS/DGPS Applications, PhD Thesis, Department of Geomatics Engineering, The University of Calgary

National Academy of Public Administration 1995, The Global Positioning System: A Shared National Asset: Recommendations for Technical Improvements and Enhancements, (Washington, D.C.: National Academy Press), p.16

Noureldin, A., Karamat, T. B., Eberts, M. D., \& El-Shafie, A. 2009, performance enhancement of MEMS-based INS/ GPS integration for low-cost navigation applications, IEEE Transactions on Vehicular Technology, 58, 10771096

Titterton, D. H. \& Weston, J. L. 2004, in Progress in Astronautics and Aeronautics, vol.207, Strapdown Inertial Navigation Technology, 2nd ed. (Reston: AIAA)

Zarchan, P. \& Musoff, H. 2005, in Progress in Astronautics and Aeronautics, vol.208, Fundamentals of Kalman Filtering: A Practical Approach, 2nd ed. (Reston: AIAA) 